Original Research Paper

\title{
Relative Transcription Expression Level of SIRT1, SIRT2 and SIRT3 in Correlation to the Expression of a Set of Selected Cancer Related Genes in Human Breast Cancer
}

\author{
${ }^{1}$ Hamdy Swelim, ${ }^{2}$ Omnia Mansour, ${ }^{3}$ Salah Abdel-Rahman, ${ }^{2}$ Mohamed Shwaireb and ${ }^{4}$ Amani Kazem \\ ${ }^{1}$ Department of Zoology, Faculty of Science, Ain Shams University, Egypt \\ ${ }^{2}$ Department of Biological and Geological science, Faculty of Education, Alexandria University, Egypt \\ ${ }^{3}$ Department of Nucleic Acid Research, Genetic Engineering and Biotechnology Research Institute, \\ City of Scientific Research and Technological Applications, Alexandria, Egypt \\ ${ }^{4}$ Department of Pathology, Medical Research Institute, University of Alexandria, Egypt
}

Article history

Received: 15-12-2017

Revised: 29-12-2017

Accepted: 18-01-2018

\section{Corresponding Author:}

Omnia Mansour

Department of Biological and

Geological Science, Faculty of

Education, Alexandria

University, Egypt

Email:

omnia_ahmed_mansour@hotmail.com

\begin{abstract}
Mammalian Sirtuins have been shown to perform distinct cellular functions and deregulated expression of these genes was reported to be involved in the development of various malignancies including breast cancer. An increasing number of evidence indicates that Sirtuins have both tumor promoter and tumor suppressor functions. However, the roles of Sirtuins have not been well-studied in breast cancer. In the present study, quantitative expression levels of Sirtuins (SIRT1-3) together with a set of cancer related genes (cMYC, P53, SOD and HIF-1 $\alpha$ genes) were assessed in malignant breast cancer and non-malignant control samples by using a high-throughput real-time PCR method. As a result, Sirtuins were found to be differentially expressed in breast cancer tissues and control samples, respectively. Particularly, expressions of SIRT1 $(\mathrm{p}=0.035)$ and SIRT3 $(\mathrm{p}=$ 0.033 ) were found to be significantly up regulated, whereas SIRT2 ( $\mathrm{p}=$ 0.032 ) gene was shown to be downregulated in breast cancer tissues compared to control samples in our study. Additionally, the expression levels of SIRT1-3 genes were correlated to both the selected cancer related genes and to clinicopathological parameters of breast cancer patients. In conclusion, SIRT1 and SIRT3 genes may act as oncogenes, whereas SIRT2 gene may operate as a tumor suppressor gene in human breast cancer.
\end{abstract}

Keywords: Breast Cancer, SIRT1, SIRT2 and SIRT3

\section{Introduction}

Breast cancer, as other cancers, has an unstable genome which generates the genetic diversity through deregulation of gene expression profiles and disruption of molecular networks (Hanahan and Weinberg, 2011). Intriguing evidence has recently emerged that genetic and epigenetic mechanisms are not separate events in cancer; they intertwine and take advantage of each other during tumorigenesis (Jones and Martienssen, 2005).

Histones acetylation is one of epigenetic marks that regulate gene expression, occurs on the $\varepsilon$-amino groups of lysine residues in the $\mathrm{N}$-terminus of the histone proteins by the opposing action of Histone Acetyltransferases (HATs) and Histone Deacetylases (HDACs) (Dong and
Cui, 2016). The deacetylation of histone tails may underlie suppression of target genes expression and heterochromatin formation (Guarente, 2000). Sirtuins, Class III Histone Deacetylases (HDACs), are a family of proteins composed of 7 members, including SIRT1-7 which are evolutionary conserved enzymes homologous with the yeast Sir2 family of proteins (Landry et al., 2000). They are Nicotinamide Adenine Dinucleotide $(\mathrm{NAD}+)$ dependent deacetylases and/or mono-Adenosine Diphosphate (ADP)-ribosyl transferases that have attracted tremendous attention as stress adaptors and posttranslational modifiers and they have been linked to many diseases including cancer (Bosch-Presegué and Vaquero; 2011). SIRT1-3 are important members of Sirtuins family. They possess efficient deacetylase activity in vitro 
and under certain extreme conditions such as chronic stress, SIRT1-3 can protect the organism by inducing cell senescence or apoptosis, they can also deacetylate a number of non-histone target proteins (Bosch-Presegué and Vaquero; 2011; He et al., 2014). SIRT1 can, in one hand, regulate multiple target proteins involved in cell cycle progression, DNA repair machinery, cell-signaling and cell metabolism (Palacios et al., 2010; Houtkooper et al., 2012). On the other hand, SIRT2 can deacetylate the $\alpha$-tubulin subunit of microtubules and it has been proposed that SIRT2 might function as a mitotic checkpoint protein in G2-M to prevent chromosomal instability, particularly in mitotic stress (North et al., 2003; Vaquero et al., 2006). While SIRT3 works as a major protein deacetylase within the mitochondrial matrix and is critical for maintaining mitochondrial integrity and function through regulation of proteins involved in metabolism, energy homeostasis, cell survival and death (Lombard et al., 2007; Hallows et al., 2011; Alhazzazi et al., 2013). Moreover, SIRT1-3 are implicated in a variety of pathological conditions including cancer but their role in cancer as oncogenes or tumor suppressor genes is still controversial and more studies are needed to further delineate their precise functions (Alhazzazi et al., 2011; Dan et al., 2012; Chen et al., 2014; Kulić et al., 2014).

Over 30000 genetic aberrations have been detected in tumor cells when compared with normal cells and according to the Cancer Gene Data Curation Project, about 4700 genes have been identified as being related to cancer (Kumar et al., 2009; Pleasance et al., 2010). The latter include tumor suppressor genes mainly P53 gene, oncogenes like Myelocytomatosis (cMYC) gene or stress adaptation genes as Superoxide Dismutase (SOD) and Hypoxia Inducible Factor-1 (HIF-1 $\alpha)$ genes (Feinberg et al., 2006).

The c-Myc oncogene is the most frequent amplified gene in human cancers and is associated with tumor aggression and poor clinical outcome. In normal cells, it promotes cell replication in response to extracellular signals, by driving quiescent cells into the cell cycle (Lin et al., 2012). In tumor cells that express high levels of c-Myc, cellular proliferation is no longer dependent on growth-factor stimulation and this uncoupling from growth factor regulation leads to the uncontrolled proliferation characteristic of cancer cells, protection against programmed cell death, loss of respect for normal tissue boundaries and metastases (Dunning et al., 1999; Lin et al., 2012). The effect of high levels of cMyc on global gene regulation is poorly understood but is widely thought to involve newly activated or repressed "Myc target genes", (Lin et al., 2012).

The P53 gene is the most frequently mutated tumor suppressor gene in cancer and P53 loss may be required for maintenance of aggressive carcinoma (Xue et al., 2007). It exists in non-stressed cells at a very low concentration due to its rapid ubiquitin-mediated degradation in proteosomes. Under stress conditions, P53 induces the transcription of various genes that are involved in cell-cycle control, apoptosis, DNA repair, differentiation and senescence (Slee et al., 2004). In cancer, its activities are inactivated and result in the loss of normal functions, especially in accurate DNA replication that leads to the proliferation of cells that are under stress conditions and tumor development (Dunning et al., 1999). An increasing number of studies indicate that a subset of mutated P53 are oncogenic and actively participate in neoplastic transformation (Weisz et al., 2007).

SOD is essential enzyme which acts as antioxidant scavenger that catalyzes the dismutation of highly reactive $\mathrm{O} 2-\bullet$ and $\mathrm{H}_{2} \mathrm{O}_{2}$ to $\mathrm{O} 2$ and less reactive $\mathrm{H}_{2} \mathrm{O}_{2}$ respectively (Halliwell, 1994) and protect cells from oxidative damage which can cause lipid peroxidation, mutagenesis and carcinogenesis. Huang et al. (2000) showed that malignant cells are highly dependent on SOD for survival that inhibition of SOD causes accumulation of cellular O2 that cause free-radical-mediated damage to mitochondrial membranes, the release of cytochrome $\mathrm{c}$ from mitochondria and apoptosis of the cancer cells.

HIF-1 is an essential transcription factor induced by reduced $\mathrm{O} 2$ availability in the cellular environment (hypoxia) and can activate over 60 direct genes, that have roles in many critical aspects of cancer biology including glycolytic metabolism, immune avoidance, angiogenesis, metastasis and therapeutic resistance (Papadakis et al., 2010; Semenza, 2010; Zhang et al., 2010). Increased HIF-1 $\alpha$ levels in diagnostic tumor biopsies are associated with increased risk of mortality in many types of tumors including breast cancer (Semenza, 2010). Recent studies have provided evidence indicating that HIF-1 mediates resistance to chemotherapy and radiation (Aebersold et al., 2001).

Cancer cell transformation has a multitude of different intertumoral genetic and epigenetic alterations (Hutchinson, 2010). The objective of the current study was therefore to elucidate in human breast cancer the role of SIRT1, SIRT2 and SIRT3 in association with the selected cancer related genes: cMYC, P53, SOD and HIF-1 $\alpha$ genes. Herein, we aimed to correlate the expression of these genes with the clinicopathological parameters of breast cancer patients.

\section{Materials and Methods}

\section{Patients and Specimens}

In total, 30 breast cancer patients have been enrolled from those admitted to the Medical Research Institute, Alexandria University, Egypt. All subjects were recruited according to the ethical rules approved by the ethical committee of the Medical Research Institute based on Belmont report. The clinicopathological prognostic characters of breast cancer patients were obtained and reviewed and reported in Table 1. 
Specimens included 30 malignant breast cancer as well as 20 adjacent non-malignant control samples freshly collected from breast cancer patient's mastectomy after surgical removal and clinical examination at the Clinical Pathology Department, Medical Research Institute, Alexandria University, Egypt. Samples were stored in RNA-later (Ambion, UK) at $-80^{\circ} \mathrm{C}$ until they were used.

\section{RNA Isolation}

Total RNA was isolated from $30 \mathrm{mg}$ of frozen tumor and control tissues using ISOLATE II RNA Mini Column Kit (Bioline, UK) according to the manufacturer's instructions. The purity and concentration of RNA samples were determined using NanoDrop spectrophotometer. Only the RNA samples that have A260/A280 ratio range from 1.8 to 2.00 were used, otherwise the RNA samples were considered contaminated. RNA samples were then stored at $-80^{\circ} \mathrm{C}$ until be used.

\section{Quantitative Real-Time Reverse-Transcription Polymerase Chain Reaction ( $q R T-P C R$ )}

The reaction and data analysis were performed according to the instructions of the thermo PikoReal ${ }^{\mathrm{TM}}$ Real-Time PCR system, using SensiFAST ${ }^{\text {TM }}$ SYBR ${ }^{\circledR}$ NoROX One-Step Kit (Bioline, UK) and specific primers. Forward and reverse primers (Qiagen, Germany) were used to target gene expressions of the following: SIRT1: Forward 5'-AAA TGC TGG CCT AAT AGA GTG G-3', reverse 5'-TGG TGG CAA AAA CAG ATA CTG A-3'; SIRT2: Forward 5'-GAA CGC TGT CGC AGA GTC ATC-3', reverse 5'-GGT TGG CTT GAA CTG CCC AG3'; SIRT3: Forward 5'-GCA TTC CAG ACT TCA GAT CGC-3', reverse 5'-GTG GCA GAG GCA AAG GTT CC3'; P53: Forward 5'-GTT CCG AGA GCT GAA TGA GG3', reverse 5'-TCT GAG TCA GGC CCT TCT GT-3'; cMYC: Forward 5'-CTT CTC TCC GTC CTC GGA TTC T-3', reverse 5'-GAA GGT GAT CCA GAC TCT GAC CTT-3'; HIF-1 $\alpha$ : forward 5'-TGG CCT TGT GAA AAA GGG T-3', reverse 5'-TTG ATG GGT GAG GAA TGG GT-3'; SOD: Forward 5'-TGT GGG GAA GCA TTA AAG G-3', reverse 5'-CCG TGT TTT CTG GAT AGA GG-3'; BActin: Forward 5'-AGA AAA TCT GGC ACC ACA CC3', reverse 5'-AGA GGC GTA CAG GGA TAG CA-3'. QRT-PCR program was applied as one cycle of cDNA synthesis at $50^{\circ} \mathrm{C}$ for $15 \mathrm{~min}$, one qRT-PCR was performed in a reaction mixture of $20 \mu \mathrm{L}$ using $10 \mu \mathrm{L}$ SensiFAST ${ }^{\mathrm{TM}}$ SYBR ${ }^{\circledR}$ No-ROX One-Step $\operatorname{Mix}(1 \mathrm{X}), 0.2 \mu \mathrm{L}$ Reverse transcriptase, $0.4 \mu \mathrm{L}$ RiboSafe RNase Inhibitor, $1.6 \mu \mathrm{L}$ forward and reverse primers (10 pm), $4 \mu \mathrm{L}$ RNA template (10 ng) and up to $16 \mu \mathrm{L}$ sterile water. The thermal cycling program included reverse transcription at $45^{\circ} \mathrm{C}$ for $10 \mathrm{~min}$, then polymerase activation at $95^{\circ} \mathrm{C}$ for 2 min followed by 40 cycles of denaturation at $95^{\circ} \mathrm{C}$ for $5 \mathrm{~s}$, annealing at $60^{\circ} \mathrm{C}$ for $10 \mathrm{~s}$ and extension at $72^{\circ} \mathrm{C}$ for $5 \mathrm{~s}$. Duplicate samples were used for data accuracy. The data for relative gene expression were analyzed by the comparative $\mathrm{Ct}$ method $\left(2^{\wedge}(-\Delta \Delta \mathrm{CT})\right)$ using $\beta$-actin as an endogenous control and gene expression in non-malignant samples as calibrator.

\section{Statistical Analysis}

All statistical analyses were done using IBM SPSS software package version 20.0 (Armonk, NY: IBM Corp). Experiments were carried out in triplicate. The Kolmogorov-Smirnov test was used to verify the normality of distribution. The comparisons between groups were determined by Mann-Whitney test. The correlations between groups were determined by Spearman's rank correlation coefficient test. Values of $\mathrm{p}<0.05$ were considered significant.

\section{Results}

\section{Clinicopathological Characteristics of Breast Cancer Patients}

The clinicopathological features of our series of Egyptian breast cancer patients are described in Table 1. All individuals underwent curative surgery at the Medical Research Institute, Alexandria University of Egypt. The mean age at diagnosis was 53.5 years, ranging from 30 to 76 years. Among these, nearly quarter of cases $(26.7 \%)$ were premenopausal while the remaining 22 cases $(73.3 \%)$ were postmenopausal. The majority of patients (28 cases out of $30,93.3 \%$ ) were suffering from Invasive Ductal Carcinoma (IDC) whereas only 2 cases $(6.7 \%)$ showed Invasive Lobular Carcinoma (ILC). Tumor size (the largest diameter) ranged from 1 to $9 \mathrm{~cm}$, patients showing tumor size $<2$ $\mathrm{cm}$ are only 3 cases $(10 \%)$; while those having tumor size included between $2-4 \mathrm{~cm}$ are 19 cases $(63.3 \%)$ and those with tumor size $>4 \mathrm{~cm}$ are 8 cases $(26.7 \%)$. Most patients had tumor grade II (20 out of $30,66.7 \%$ ) where only 10 patients $(33.3 \%)$ had tumor grade III. The frequency of positive lymph node metastases was $63.3 \%$ (19 patients out of 30). The profile of ER $\alpha$ and HER2 as determined by immunohistochemistry was positive in 24 and 18 patients $(80 \%$ and $60 \%)$ respectively based on the $10 \%$ cut off level for expression analysis.

The Relative Expression Level of SIRT1, SIRT2 and SIRT3 and Selected Cancer Related Genes: cMYC, P53, SOD and HIF-1 $\alpha$, in Human Breast Cancer Samples

The qRT-PCRT technique was used to quantify the relative transcription expression level of SIRT1, SIRT2, SIRT3, cMYC, P53, SOD and HIF-1 $\alpha$ genes in malignant and non-malignant samples of human breast cancer (Fig. 1). 


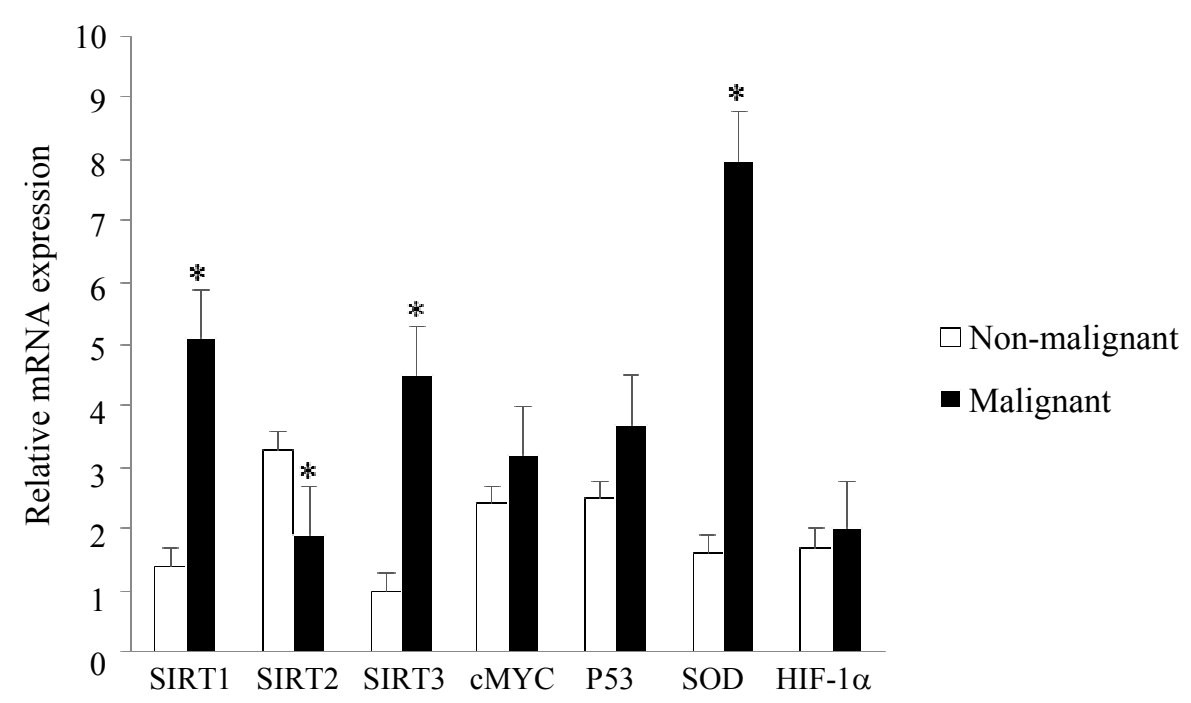

Fig. 1: Real-time PCR experiments showing differential gene expression associated with human breast samples, as compared to their corresponding controls $(* p<0.05)$

Table 1: Clinicopathological prognostic data of breast cancer patients

\begin{tabular}{|c|c|}
\hline \multirow{2}{*}{\multicolumn{2}{|c|}{$\frac{\text { Parameters }}{\text { Age (vears) }}$}} \\
\hline & \\
\hline Min-Max & $30-76$ \\
\hline Mean \pm SD & $53.5 \pm 11.5$ \\
\hline Median age & 52 \\
\hline \multicolumn{2}{|l|}{ Menopausal status } \\
\hline Pre & $8(26.7)$ \\
\hline Post & $22(73.3)$ \\
\hline \multicolumn{2}{|l|}{ Histological type } \\
\hline Invasive lobular carcinoma (ILC) & $2(6.7)$ \\
\hline Invasive ductal carcinoma (IDC) & $28(93.3)$ \\
\hline \multicolumn{2}{|l|}{ Tumor size $(\mathrm{T})(\mathrm{cm})$} \\
\hline $\operatorname{Min}-\operatorname{Max}$ & $1-9$ \\
\hline$<2$ & $3(10)$ \\
\hline $2-4$ & $19(63.3)$ \\
\hline$>4$ & $8(26.7)$ \\
\hline \multicolumn{2}{|l|}{ Tumor grade } \\
\hline II & $20(66.7)$ \\
\hline III & $10(33.3)$ \\
\hline \multicolumn{2}{|l|}{ Lymph node metastasis } \\
\hline Negative & $11(36.7)$ \\
\hline \multicolumn{2}{|l|}{ Positive } \\
\hline $1(1-3)$ & $7(23.3)$ \\
\hline $2(4-9)$ & $5(16.7)$ \\
\hline $3(\geq 10)$ & $7(23.3)$ \\
\hline \multicolumn{2}{|l|}{$\mathrm{ER} \alpha$} \\
\hline Negative & $6(20)$ \\
\hline \multicolumn{2}{|l|}{ Positive } \\
\hline+1 & $2(6.7)$ \\
\hline+2 & $12(40)$ \\
\hline+3 & $10(33.3)$ \\
\hline \multicolumn{2}{|l|}{ HER2 } \\
\hline Negative & $12(40)$ \\
\hline \multicolumn{2}{|l|}{ Positive } \\
\hline+1 & $10(33.3)$ \\
\hline+2 & $6(20)$ \\
\hline+3 & $2(6.7)$ \\
\hline
\end{tabular}

In our experiment, SIRT1 and SIRT3 genes were found to be upregulated in 76.7 and $63.6 \%$ of malignant samples, respectively; whereas, SIRT2 gene was found to be downregulated in $62.1 \%$ of malignant tissues. The upregulation of both SIRT1 $(5.1 \pm 1.5$, mean \pm SEM, $p=$ $0.035)$ and SIRT3 genes $(4.5 \pm 1, p=0.033)$ as well as the downregulation of SIRT2 gene $(1.9 \pm 0.48$, mean \pm SEM, $p=0.032$ ) were found to be significant in malignant samples compared to non-malignant samples of human breast cancer.

On the other hand, 33.3, 55.6, 66.6 and $42.3 \%$ of malignant samples had over-expression of cMYC, P53, SOD and HIF-1 $\alpha$ genes, respectively. Malignant tissues had non-significant high level of mRNA expression of cMYC (3.2 \pm 1.3 , mean \pm SEM, $p=0.228)$, P53(3.7 \pm 1.1 , $\mathrm{p}=0.690)$ and HIF-1 $\alpha(1.7 \pm 0.57, \mathrm{p}=0.428)$ genes than those in non-malignant samples $(2.4 \pm 0.64,2.5 \pm 0.8$, $2.0 \pm 0.68$ respectively). However, there was significant high level of SOD gene expression in malignant tissues (7.97 $\pm 3.9, p=0.046)$, compared to non-malignant samples (1.6 \pm 0.4$)$ of human breast cancer.

The correlation between SIRT1, SIRT2 and SIRT3 and a set of selected cancer related genes: cMYC, P53, SOD and HIF- $1 \alpha$, in human breast cancer samples

In regard to the correlations between the relative transcription expression levels of genes included in this study (Fig. 2), Spearman's rank correlation showed a significant positive correlation between SIRT1 and P53 $(\mathrm{P}=0.012)$, SIRT3 and $\mathrm{cMYC}(\mathrm{P}=0.010), \mathrm{cMYC}$ and $\mathrm{P} 53(\mathrm{p}=0.000)$ and SOD and HIF-1 $\alpha(\mathrm{P}=0.012)$ genes.

On the other side, there were no significant correlations between the expression levels of each of the following pairs: SIRT1 and SIRT2 $(\mathrm{p}=0.732), \operatorname{SIRT} 1$ and SIRT3 ( $\mathrm{p}$ $=0.331)$ and SIRT2 and SIRT3 $(\mathrm{p}=0.691)$, cMYC and SOD $(p=0.823)$, cMYC and HIF-1 $\alpha(p=0.491)$, P53 and $\operatorname{SOD}(\mathrm{p}=0.301)$ and P53 and HIF- $1 \alpha(\mathrm{p}=0.195)$ genes. 

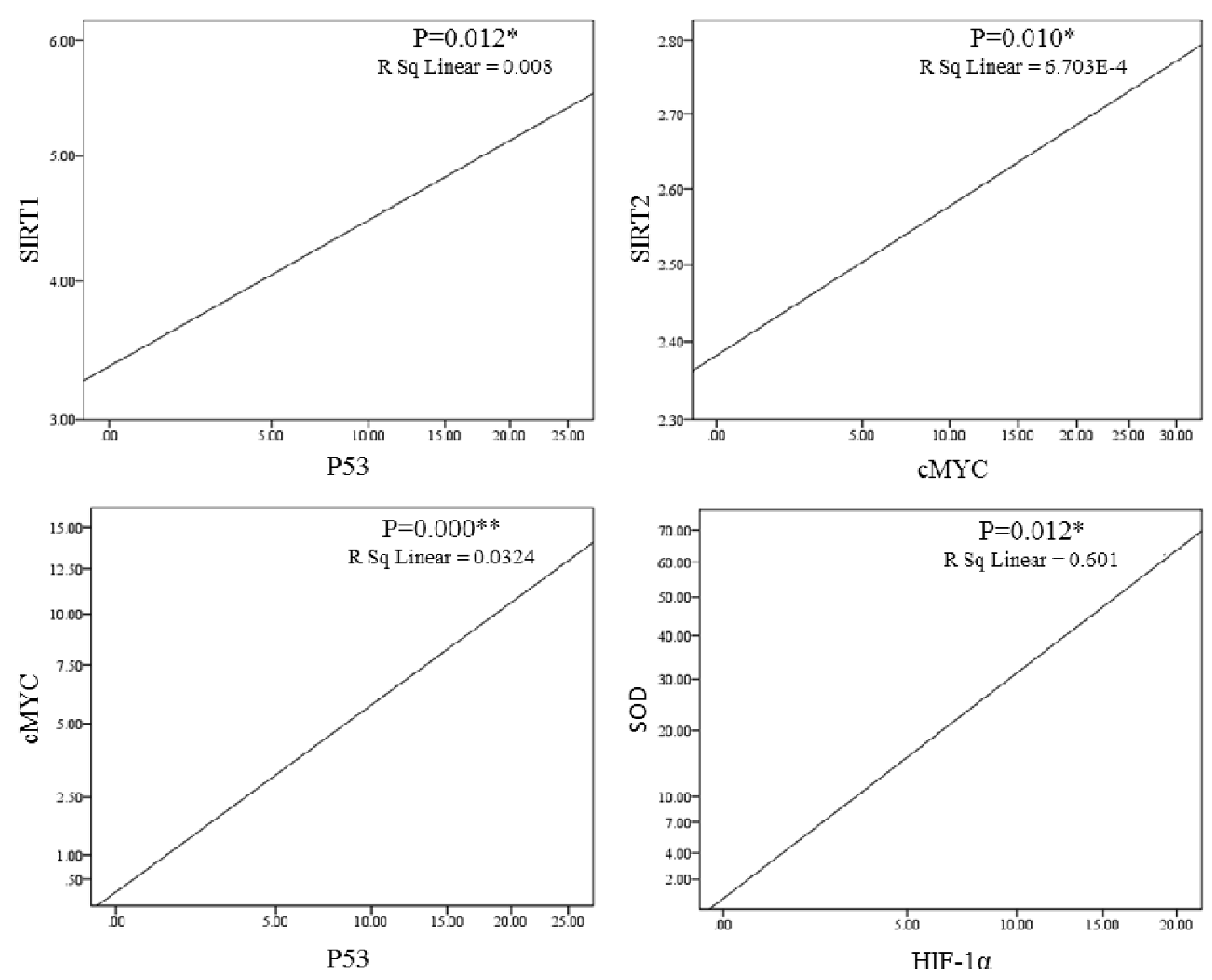

Fig. 2: Scatter plots showing the significant correlations between the relative expression levels of a pair of particular genes included in our study $(* \mathrm{p}<0.05 ; * * \mathrm{p}<0.01)$

Table 2: The correlation between the relative transcription expression levels of cMYC, P53, SOD and HIF-1 $\alpha$; SIRT1, SIRT2 and SIRT3 and the clinicopathological parameters of breast cancer patients

\begin{tabular}{|c|c|c|c|c|c|c|c|c|}
\hline & & \multicolumn{7}{|c|}{ Relative gene expression } \\
\hline \multicolumn{2}{|l|}{ Pathological parameters } & SIRT1 & SIRT2 & SIRT3 & $\mathrm{cMYC}$ & P53 & SOD & HIF- $1 \alpha$ \\
\hline \multirow[t]{2}{*}{ Age } & rs & 0.259 & 0.262 & 0.262 & 0.352 & $0.470 *$ & 0.062 & 0.279 \\
\hline & $\mathrm{p}$ & 0.284 & 0.279 & 0.388 & 0.152 & $0.049 *$ & 0.820 & 0.296 \\
\hline \multirow[t]{2}{*}{ Menopausal status } & rs & 0.175 & -0.065 & 0.089 & 0.155 & 0.299 & 0.226 & 0.156 \\
\hline & $\mathrm{p}$ & 0.475 & 0.790 & 0.772 & 0.538 & 0.228 & 0.400 & 0.563 \\
\hline \multirow[t]{2}{*}{ Histological type } & rs & 0.071 & 0.081 & -0.050 & -0.054 & -0.018 & 0.120 & -0.233 \\
\hline & $\mathrm{p}$ & 0.711 & 0.675 & 0.826 & 0.787 & 0.928 & 0.558 & 0.253 \\
\hline \multirow[t]{2}{*}{ Tumor size } & rs & -0.304 & -0.068 & $0.453 *$ & -0.324 & -0.279 & 0.106 & 0.048 \\
\hline & $\mathrm{p}$ & 0.103 & 0.726 & $0.034^{*}$ & 0.100 & 0.159 & 0.607 & 0.815 \\
\hline \multirow[t]{2}{*}{ Tumor grade } & rs & 0.148 & 0.079 & -0.391 & 0.058 & $0.474 *$ & $0.427^{*}$ & $0.501 *$ \\
\hline & $\mathrm{p}$ & 0.453 & 0.696 & 0.088 & 0.784 & $0.017^{*}$ & $0.037^{*}$ & $0.013 *$ \\
\hline \multirow[t]{2}{*}{ Vascular invasion } & rs & -0.193 & 0.060 & 0.136 & -0.067 & -0.268 & -0.142 & -0.270 \\
\hline & $\mathrm{p}$ & 0.308 & 0.758 & 0.547 & 0.740 & 0.177 & 0.489 & 0.182 \\
\hline \multirow[t]{2}{*}{ Lymph node metastasis } & rs & -0.168 & -0.097 & 0.117 & -0.292 & -0.370 & -0.313 & -0.344 \\
\hline & $\mathrm{p}$ & 0.374 & 0.618 & 0.605 & 0.139 & 0.058 & 0.120 & 0.085 \\
\hline \multirow[t]{2}{*}{ ER } & rs & -0.450 & 0.443 & 0.099 & 0.191 & -0.035 & 0.526 & -0.366 \\
\hline & $\mathrm{p}$ & 0.080 & 0.098 & 0.747 & 0.513 & 0.905 & 0.079 & 0.241 \\
\hline \multirow[t]{2}{*}{ HER2 } & rs & 0.026 & 0.106 & $0.729 * *$ & 0.135 & -0.115 & -0.251 & 0.381 \\
\hline & $\mathrm{p}$ & 0.926 & 0.719 & $0.007 * *$ & 0.660 & 0.709 & 0.456 & 0.247 \\
\hline
\end{tabular}

rs: Spearman coefficient; *: Statistically significant at $\mathrm{p}<0.05 ; * *$ : Statistically significant at $\mathrm{p}<0.01$ 
The correlations between the relative transcription expression levels ofSIRT1, SIRT2 and SIRT3 and the selected cancer related genes: cMYC, P53, SOD and HIF-1 $\alpha$, together with the clinicopathological parameters of breast cancer patients

In regard to the correlation between genes expression and the clinicopathological features of breast cancer patients (Table 2), Spearman's rank correlation showed that there were positive correlations between the relative transcription expression level of SIRT3 gene and both tumor size $(p=0.034)$ and HER2 status $(p=0.007)$, P53 gene and both patient's ages $(\mathrm{p}=0.049)$ and tumor grade $(p=0.017)$ and both SOD and HIF-1 $\alpha$ genes and tumor grade ( $p=0.037$ and $p=0.013$, respectively).

On the other hand, there was no significant correlation between the relative transcription expression of SIRT1, SIRT2 and cMYC and any of the clinicopathological parameters of breast cancer patients.

\section{Discussion}

Breast cancer, like other cancer types, has thousands of genetic aberrations that enable tumor formation and progression (Kumar et al., 2009; Pleasance et al., 2010). The genetic path to cancer is not related only to mutation of oncogenes or tumor suppressor genes, but also to intracellular stress adaptation and/or abnormal expression due to epigenetics alterations (Sharma et al., 2010). SIRT1-3, members of Class III histone deacetylases of sirtuin family, are related to epigenetic regulatory proteins that are capable of deacetylation not only chromatin proteins, which are key elements in the regulation of gene expression, but also of non-histone proteins leading to inappropriate activation or inhibition of various cellular signaling pathways (Minucci and Pelicci, 2006; Sandoval and Esteller, 2012). However, the efficiency and physiological relevance of their activity are not known and their role in cancer still controversial (Kulić et al., 2014; Teng et al., 2014). In our study, we aimed to investigate the mRNA expressions of SIRT1-3 genes and a set of cancer related genes like cMYC, P53, SOD and HIF-1 $\alpha$ in human breast cancer. To the best of our knowledge, this is the first report that investigates the expression of this set of genes together in human breast cancer.

\section{Genes Expression in Human Breast Cancer Samples}

SIRT1 is detected in many types of cancers, but its possible role in cancer has posed a dilemma (Kulić et al., 2014). Yuan et al. (2013) suggested that SIRT1 has a dual role in the development of tumors as a tumor suppressor or promoter depending on the type of tumor and the spatial distribution of SIRT1 upstream and downstream factors. In breast cancer, Wang et al. (2008) showed that SIRT1gene expression is reduced and it acts as a tumor suppressor gene, while Ashraf et al. (2006) showed that there was no association between SIRT1 gene expression and breast cancer. In our study, we found a significant increase in SIRT1 gene expression $(p=0.035)$ in malignant samples and this finding supports other reports that SIRT1may act as an oncogene and contribute to tumor development in breast cancer (Cao et al., 2014; Santolla et al., 2015).

SIRT2 upregulation or downregulation is detected in many cancer types (Hiratsuka et al., 2003; Dan et al., 2012; Chen et al., 2013; Liu et al., 2013; Ming et al., 2014). Ashraf et al. (2006) showed that there was no significant difference in SIRT2 gene expression between breast cancer biopsies and normal breast tissue. In our study, there was a significantly low SIRT2 gene expression in malignant samples of breast cancer patients $(p=0.032)$ and this support the claim that SIRT2 may function as a tumor suppressor by maintaining cellular mitotic integrity and its dysfunction leads to genetic instability and tumorigenesis (Hiratsuka et al., 2003; Kim et al., 2011).

Additionally, the deregulation of SIRT3 expression has been observed in different cancers (Huang et al., 2014; Liu et al., 2014; Yan et al., 2014). Chen et al. (2014) mentioned that SIRT3 can function either as a tumor promoter or suppressor depending on tumor type, cellular stresses or cell death stimuli. In breast cancer, Ashraf et al. (2006) found no significant differences in SIRT3 gene expression between malignant and normal breast biopsies. However, our finding support other results reporting (He et al., 2014) that there was a significant high level of SIRT3 gene expression in breast cancer patients $(p=0.033)$. Thus, SIRT3 could function as a tumor promoter and plays a prosurvival role in cancer (Alhazzazi et al., 2011).

Our data reported that $33.3 \%$ of the tumor samples had overexpression of cMYC gene, however, there was no significant difference in cMYC gene expression between the malignant and non-malignant samples in breast cancer $(p=0.235)$. This is in agreement with studies reporting that $\mathrm{cMYC}$ overexpression at the mRNA level is in 22 to $35 \%$ of breast tumors (Bieche et al., 1999; Scorilas et al., 1999).

Moreover, an increasing number of studies indicate that a subset of mutated P53 are oncogenic and actively participate in neoplastic transformation (Weisz et al., 2007). Our results have shown that $55.6 \%$ of the tumor samples had P53 overexpressioneven though there was no significant differences in P53expression between malignant and non-malignant samples in breast cancer $(\mathrm{p}=0.690)$.

In the meantime, a defect in SOD is experimentally proved to be associated with several types of cancer such as hepatocellular carcinoma and brain tumor (Elchuri et al., 2005; Aggarwal et al., 2006). Previous investigators 
have measured the enzymatic activities of the SODs in breast cancers. Huang et al. (2007) suggested that SOD can act as a tumor suppressor by decreasing growth and survival of breast cancer cells. On the other hand, some studies have reported a correlation between high SOD level and invasiveness of breast cancer (Tsanou et al., 2004; Kattan et al., 2008). Er et al. (2004) speculated that upregulation of SOD expression induced by oxidative stress or local inflammation may contribute a selective growth advantage to tumor cells compared to their normal counterparts (Khan et al., 2010). In agreement to other studies (Tas et al., 2005; Rajneesh et al., 2008), our findings have shown that $66.6 \%$ of the tumor samples had overexpression of SOD gene and that there was a significant difference in the expression levels of this gene in malignant versus non-malignant samples $(p=0.046)$.

Interestingly, all experimental data indicate that HIF1 is over-expressed in many human cancers, mainly in the earliest detected neoplastic lesions (Talks et al., 2000; Mandriota et al., 2002). Our data described $42.3 \%$ of the tumor samples with HIF-1 $\alpha$ overexpression but no significant difference between malignant and non-malignant breast cancer samples ( $p$ $=0.428$ ) have been reported.

\section{Correlations between Different Genes in Human Breast Cancer Samples}

The correlation between the expression levels of SIRT1-3 and cancer related genes have been shown to be significantly positive between SIRT1 and P53 genes ( $p=$ 0.012). This is in accordance with Wilking and Ahmad (2015) who reported that P53 positively regulates the transcription of SIRT1 and promotes its activity. In a feedback loop, overexpression of SIRT1 would lead to its own repression via P53 acetylation and inactivation. Kozako et al. (2014) showed that in response to DNA damage and oxidative stress, SIRT1 deacetylate and inactivate P53 and blocks its nuclear translocation, leading to the accumulation of P53 in both the cytosol and mitochondria. Thus, we suggest that inhibition of the function of P53 protein by SIRT1 may induce the cell to produce more P53 to compensate its function and this supports the oncogenic consequences of SIRT1 overexpression. Interestingly, SIRT3 gene expression was significantly correlated with the expression of cMYC oncogene $(P=0.010)$ in this study. This finding supports the idea that SIRT3 can act as a tumor promoter and can have oncogenic consequences in breast cancer. Moreover, there was in this study a significant positive correlation between the expression levels of cMYC and P53 genes $(p=0.000)$ as previously observed in lymphoma, leukemia, non-small cell lung carcinoma and hepatocellular carcinoma (Gaidano et al., 1991; Morkve et al., 1992; Kawate et al., 1999). c-Myccan transactivate the P53 promoter and thus may induce expression of P53 (Reisman et al., 1993), mutant P53 protein is capable of transactivating c-mycpromoter, or loss of wild-type P53 alleles is coupled closely with induction of gene amplification in some cells (Kawate et al., 1999). Thus, inactivation of P53 in tumors with deregulated Myc expression may be one mechanism by which cells have evaded control of tumorigenesis by cell death. We also found a significant positive correlation between the expression of SOD and HIF-1 $\alpha$ genes $(\mathrm{P}=0.012)$. There are multiple sources of reactive oxygen in tumors and SOD can act as antioxidant scavenger thus these can clearly influence HIF1 activity in a hypoxia-independent way (López-Lázaro, 2007; Dewhirst et al., 2008).

\section{Correlations between Genes Expression and Clinicopathological Parameters of Breast Cancer Patients}

Concerning the correlation between SIRT1-3, cancer related genes and the clinicopathological data of the patients, we observed significant positive correlations between the relative transcription levels of SIRT3 and both tumor size $(p=0.034)$ and HER2 status $(p=0.007)$; between the relative transcription levels of P53 gene and both patient's ages $(p=0.049)$ and tumor grade $(p=$ $0.017)$; and between tumor grade and the relative transcription levels of both SOD $(p=0.037)$ and HIF- $1 \alpha$ $(p=0.013)$. Together, these results might mean that SIRT3 abnormalities are early events in breast tumorigenesis (Desouki et al., 2014) and have a role in tumor progression, that P53 deregulation by mutation or inhibition is frequently associated with tumor progression (Fearon and Vogelstein 1990; Sidransky et al., 1992; Kemp et al., 1993) and finally that overexpression of SOD and HIF-1 $\alpha$ genes can support cancer cell progression against oxidative stress and hypoxic conditions.

\section{Conclusion}

Briefly, SIRT1 gene overexpression can have an oncogenic function in human breast cancer and can promote P53 gene expression through inactivation of P53 protein. Thus, P53 overexpression is stimulating to compensate its function and this may be one mechanism by which tumor cells evade control of cell death. Additionally, SIRT3 gene overexpression can act as an oncogene and its role is not only limited to tumor promotion but extends to play a role in tumor progression as well. On the other hand, SIRT2 gene expression is downregulated and can act as a tumor suppressor gene in human breast cancer. Our data reported that cMyc gene expression stimulates both SIRT3 and P53 overexpression and that SOD overexpression supports tumor formation and has a role in human breast cancer. In the meantime, SOD overexpression can stimulate HIF- $1 \alpha$ expression through 
$\mathrm{H}_{2} \mathrm{O}_{2}$ production and this can influence HIF-1 activity in a hypoxia independent way. To summarize, SIRT1-3 can act not only as epigenetic markers but can have a direct important role in human breast cancer through their interaction with other genes.

\section{Acknowledgment}

We are grateful to Associate Prof. Abeer El Wakil, Dept. Molecular Biology, Umeå University, Sweden, for her advices in practical work and for reviewing the manuscript.

\section{Author's Contributions}

All authors contributed equally in this work.

\section{Ethics}

The authors declare that this article is original and corresponds to the ethical norms specified by the Online Journal of Biological Sciences.

\section{Conflict of Interest Statement}

The authors have no conflicts of interest to disclose.

\section{References}

Aebersold, D.M., P. Burri, K.T. Beer, J. Laissue and R.H. Greiner et al., 2001. Expression of hypoxiainducible factor-1 $\alpha$ : A novel predictive and prognostic parameter in radiotherapy of oropharyngeal cancer. Cancer Res., 61: 2911-2916.

Aggarwal, S., M. Subberwal, S. Kumar and M. Sharma, 2006. Brain tumor and role of beta-carotene, atocopherol, superoxide dismutase and glutathione peroxidases. J. Cancer Res., 2: 24-27. DOI: $10.4103 / 0973-1482.19771$

Alhazzazi, T.Y., P. Kamarajan, E. Verdin and Y.L. Kapila, 2011. SIRT3 and cancer: Tumor promoter or suppressor? Biochim. Biophys. Acta., 1816: 80-88. DOI:10.1002/cncr.25676

Alhazzazi, T.Y., P. Kamarajan, E. Verdin and Y.L. Kapila, 2013. Sirtuin-3 (SIRT3) and the Hallmarks of Cancer. Genes Cancer, 4: 164-171. DOI: $10.1177 / 1947601913486351$

Ashraf, N., S. Zino, A. Macintyre, D. Kingsmore and A.P. Payne et al., 2006. Altered sirtuin expression is associated with node-positive breast cancer. Br. J. Cancer, 95: 1056-61. DOI: 10.1038/sj.bjc.6603384

Bieche, I., I. Laurendeau, S. Tozlu, M. Olivi and D. Vidaud et al., 1999. Quantitation of MYC gene expression in sporadic breast tumors with a realtime reverse transcription-PCR assay. Cancer Res., 59: 2759-2765.
Bosch-Presegué, L. and A. Vaquero, 2011. The dual role of sirtuins in cancer. Genes Cancer, 2: 648-662. DOI: $10.1177 / 1947601911417862$

Cao, Y., W. Li, G. Wan, Y. Li and X. Du et al., 2014. Correlation and prognostic value of SIRT1 and Notch1 signaling in breast cancer. J. Exp. Clin. Cancer Res., 33: 97. DOI: $10.1186 / \mathrm{s} 13046-014-0097-2$

Chen, J., A.W. Chan, K.F. To, W. Chen and Z. Zhang et al., 2013. SIRT2 over expression in hepatocellular carcinoma mediates epithelial to mesenchymal transition by protein kinase $\mathrm{B} /$ glycogen synthase kinase-3b/b-catenin signaling. Hepatology, 57: 2287-2298. DOI: 10.1002/hep.26278.

Chen, Y., L.L. Fu, X. Wen, X.Y. Wang and J. Liu et al., 2014. Sirtuin-3 (SIRT3), a therapeutic target with oncogenic and tumor-suppressive function in cancer. Cell Death Disease, 5: e1047-e1047.

DOI: $10.1038 /$ cddis.2014.14

Dan, L., O. Klimenkova, M. Klimiankou, J.H. Klusman and M.M. Heuvel-Eibrink et al., 2012. The role of sirtuin 2 activation by nicotinamide phosphoribosyl transferase in the aberrant proliferation and survival of myeloid leukemia cells. Haematologica, 97: 551-559. DOI: 10.3324/haematol.2011.055236

Desouki, M.M., I. Doubinskaia, D. Gius and S.A. Abdulkadir, 2014. Decreased mitochondrial SIRT3 expression is potential molecular biomarker associated with poor outcome in breast cancer. Hum. Pathol., 45: 1071-1077. DOI: 10.1016/j.humpath.2014.01.004

Dewhirst, M.W., Y. Cao and B. Moeller, 2008. Cycling hypoxia and free radicals regulate angiogenesis and radiotherapy response. Nature Rev. Cancer, 8: 425-437. DOI: 10.1038/nrc2438

Dong, Z. and H. Cui, 2016. Function of sirtuins in cancer stem cells. Int. J. Stem Cell Res., 3: 024-024. DOI: $10.23937 / 2469-570 X / 1410024$

Dunning, A.M., C.S. Healey, P.D. Pharoah, M.D. Teare and B.A. Ponder et al., 1999. A systematic review of genetic polymorphisms and breast cancer risk. Cancer Epidemiol. Biomarkers, 8: 843-854.

Elchuri, S., T.D. Oberley, W. Qi, R.S. Eisenstein and R.L. Jackson et al., 2005. CuZnSOD deficiency leads to persistent and widespread oxidative damage and hepatocarcinogenesis later in life. Oncogene, 24: 367-380. DOI: 10.1038/sj.onc. 1208207

Er, T.K., M.F. Hou, E.M. Tsa, J.N. Lee and L.Y. Tsai, 2004. Differential expression of manganese containing superoxide dismutase in patients with breast cancer in Taiwan. Ann. Clin. Lab Sci., 34: 159-64.

Fearon, E.R. and B. Vogelstein, 1990. A genetic model for colorectal tumorigenesis. Cell, 61: 759-767. DOI: 10.1016/0092-8674(90)90186-I 
Feinberg, A.P., R. Ohlsson and S. Henikoff, 2006. The epigenetic progenitor origin of human cancer. Nat. Rev. Genet., 7: 21-33. DOI: 10.1038/nrg1748

Gaidano, G., P. Ballerini, J.Z. Gong, G. Inghirami and A. Neri et al., 1991. p53 mutation in human lymphoid malignancies: Association with Burkitt lymphoma and chronic lymphocytic leukemia. Proc. Natl. Acad. Sci. USA, 88: 5413-5417.

Guarente, L., 2000. Sir2 links chromatin silencing, metabolism and aging. Genes Dev., 14: 1021-1026. DOI:10.1101/gad.14.9.1021

Halliwell, B., 1994. Free radicals, antioxidants and human disease. Curiosity, cause, or consequence? Lancet., 344: 721-724. DOI: $10.1016 / \mathrm{S} 0140-6736(94) 92211-\mathrm{X}$

Hallows, W.C., W. Yu, B.C. Smith, M.K. Devries and J.J. Ellinger et al., 2011. Sirt3 promotes the urea cycle and fatty acid oxidation during dietary restriction. Mol. Cell, 41: 139-149.

DOI: 10.1016/j.molcel.2011.01.002

Hanahan, D. and R.A. Weinberg, 2011. Hallmarks of cancer: The next generation. Cell, 144: 646-74. DOI: $10.1016 /$ j.cell.2011.02.013

He, S., C. He, H. Yuan, S. Xiong and Z. Xiao et al., 2014. The SIRT 3 expression profile is associated with pathological and clinical outcomes in human breast cancer patients. Cell Physiol. Biochem., 34: 2061-2069. DOI: 10.1159/000366401

Hiratsuka, M., T. Inoue, T. Toda, N. Kimura and Y. Shirayoshi et al., 2003. Proteomics-based identification of differentially expressed genes in human gliomas: Down regulation of SIRT2 gene. Biochem. Biophys. Res. Commun., 309: 558-566. DOI: $10.1016 /$ j.bbrc.2003.08.029

Houtkooper, R.H., E. Pirinen and J. Auwerx, 2012. Sirtuins as regulators of metabolism and healthspan. Nat. Rev. Mol. Cell Biol., 13: 225-238. DOI: $10.1038 / \mathrm{nrm} 3293$

Huang, K.H., C.C. Hsu, W.L. Fang, C.W. Chi and M.T. Sung et al., 2014. SIRT3 expression as a biomarker for better prognosis in gastric cancer. World $\mathrm{J}$. Surgery, 38: 910-917. DOI: 10.1007/s00268-0132359-0

Huang, P., L. Feng, E.A. Oldham, M.J. Keating and W. Plunkett, 2000. Superoxide dismutase as a target for the selective killing of cancer cells. Nature, 407: 390-395. DOI: 10.1038/35030140

Huang, Y.S., W.J. Su, Y.H. Huang, C.Y. Chen and F.Y. Chang et al., 2007. Genetic polymorphisms of manganese superoxide dismutase, NAD $(\mathrm{P}) \mathrm{H}$ : Quinone oxidoreductase, glutathione S-transferase $\mathrm{M} 1$ and $\mathrm{T} 1$ and the susceptibility to drug-induced liver injury. J. Hepatol., 47: 128-134.

DOI: $10.1016 /$ j.jhep.2007.02.009
Hutchinson, L., 2010. Breast cancer: Challenges, controversies, breakthroughs. Nat. Rev. Clin. Oncol., 7: 669-670.

DOI: $10.1038 /$ nrclinonc. 2010.192

Jones, P.A. and R. Martienssen, 2005. A blueprint for a human epigenome project: The AACR human epigenome workshop. Cancer Res., 65: 1124111246. DOI: 10.1158/0008-5472.CAN-05-3865

Kattan, Z., V. Minig, P. Leroy, M. Dauca and P. Becuwe, 2008. Role of manganese superoxide dismutase on growth and invasive properties of human estrogen-independent breast cancer cells. Breast Cancer Res. Treat., 108: 203-215. DOI: $10.1007 / \mathrm{s} 10549-007-9597-5$

Kawate, S., T. Fukusato, S. Ohwada, A. Watanuki and Y. Morishita, 1999. Amplification of c-myc in Hepatocellular carcinoma: Correlation with clinicopathologic features, proliferative activity and p53 overexpression. Oncology, 57: 157-163. DOI: $10.1159 / 000012024$

Kemp, C.J., L.A. Donehower, A. Bradley and A. Balmain, 1993. Reduction of p53 gene dosage does not increase initiation or promotion but enhances malignant progression of chemically induced skin tumors. Cell, 74: 813-822. DOI: 10.1016/0092-8674(93)90461-X

Khan, M.A., M. Tania, D. Zhang and H. Chen, 2010. Antioxidant enzymes and cancer. Chin. J. Cancer Res., 22: 87-92. DOI: $10.1007 / \mathrm{s} 11670-010-0087-7$

Kim, H.S., A. Vassilopoulos, R.H. Wang, T. Lahusen and Z. Xiao et al., 2011. Sirt2 maintains genome integrity and suppresses tumorigenesis through regulating apc/c activity. Cancer Cell, 20: 487-499. DOI: $10.1016 /$ j.ccr.2011.09.004

Kozako, T., T. Suzuki, M. Yoshimitsu, N. Arima and S. Honda et al., 2014. Anticancer agents targeted to sirtuins. Molecules, 19: 20295-20313. DOI: $10.3390 /$ molecules 191220295

Kulić, A., M. Sirotković-Skerlev, N. DedićPlavetić, B. Belev and S. Kralik-Oguić et al., 2014. Sirtuins in tumorigenesis. Period Boil., 116: 381-386.

Kumar, G.R., T.K. Subazini, K. Subha, C.P. Rajadurai and L. Prabakar, 2009. Can Gene Base (CGB)-a database on cancer related genes. Bioinformation, 3 : 422-424.

Landry, J., A. Sutton, S.T. Tafrov, R.C. Heller and J. Stebbins et al., 2000. The silencing protein sir2 and its homologs are nad-dependent protein deacetylases. Proc. Natl. Acad. Sci. USA, 97: 5807-5811. DOI: $10.1073 /$ pnas. 110148297

Liu, C., Z. Huang, H. Jiang and F. Shi, 2014. The sirtuin 3 expression profile is associated with pathological and clinical outcomes in colon cancer patients. Biomed. Res. Int., 2014: 87126-87126. DOI: $10.1155 / 2014 / 871263$ 
Lin, C.Y., J. Lovén, P.B. Rahl, R.M. Paranal and C.B. Burge et al., 2012. Transcriptional amplification in tumor cells with elevated c-Myc. Cell, 151: 56-67. DOI: $10.1016 /$ j.cell.2012.08.026

Liu, P.Y., N. Xu, A. Malyukova, C.J. Scarlett and Y.T. Sun et al., 2013. The histone deacetylase SIRT2 stabilizes Myconcoproteins. Cell Death Different., 20: 503-514. DOI:10.1038/cdd.2012.147

Lombard, D.B., F.W. Alt, H.L. Cheng, J. Bunkenborg and R.S. Streeper et al., 2007. Mammalian Sir2 homolog SIRT3 regulates global mitochondrial lysine acetylation. Mol. Cell Biol., 24: 8807-8814. DOI: 10.1128/MCB.01636-07

López-Lázaro, M., 2007. Dual role of hydrogen peroxide in cancer: Possible relevance to cancer chemoprevention and therapy. Cancer Lett., 252: 1-8. DOI: 10.1016/j.canlet.2006.10.029

Mandriota, S.J., K.J. Turner, R.D. Davies, P.G. Murray and N.V. Morgan et al., 2002. HIF activation identifies early lesions in VHL kidneys: Evidence for site-specific tumor suppressor function in the nephron. Cancer Cell, 1: 459-468.

DOI: 10.1016/S1535-6108(02)00071-5

Ming, M., L. Qiang, B. Zhao and Y.Y. He, 2014. Mammalian SIRT2 inhibits keratin 19 expression and is a tumor suppressor in skin. Exp. Dermatol., 23: 207-209. DOI: 10.1111/exd.12323

Minucci, S. and P.G. Pelicei, 2006. Histone deacelylase inhibitors and file promise of" epigenelic (and more) treatments for cancer. Nat. Rev. Cancer, 6: 38-51. DOI: $10.1038 / \mathrm{nrc} 1779$

Morkve, O., O.J. Halvorsen, L. Stangeland, A. Gulsvik and O.D. Laerum, 1992. Laerum Quantitation of biological tumor markers (p53, myc, Ki-67 and DNA ploidy) by multiparameter flow cytometry in nonsmall cell lung cancer. Int. J. Cancer, 52: 851-855. DOI: $10.1002 /$ ijc.2910520603

North, B.J., B.L. Marshall, M.T. Borra, J.M. Denu and E. Verdin, 2003. The human Sir2 ortholog, SIRT2, is an NAD+-dependent tubulin deacetylase. Mol. Cell, 11: 437-444. DOI: $10.1016 / \mathrm{S} 1097-2765(03) 00038-8$

Palacios, J.A., D. Herranz, M.L. De Bonis, S. Velasco and M. Serrano et al., 2010. SIRT1 contributes to telomere maintenance and augments global homologous recombination. J. Cell Biol., 191: 1299-313.

DOI: $10.1083 /$ jcb.201005160

Papadakis, A.I., E. Paraskeva, P. Peidis, H. Muaddi and S. Li et al., 2010. eIF2\{alpha\} Kinase PKR modulates the hypoxic response by Stat3-dependent transcriptional suppression of HIF-1 \{alpha\}. Cancer Res., 70: 7820-7829.

DOI: 10.1158/0008-5472.CAN-10-0215
Rajneesh, C.P., A. Manimaran, K.R. Sasikala and P. Adaikappan, 2008. Lipid peroxidation and antioxidant status in patients with breast cancer. Singapore Med. J., 49: 640-643

Reisman, D., N.B. Elkind, B. Roy, J. Beamon and V. Rotter, 1993. c-Myc trans-activates the p53 Promoter through a required downstream CACGTG Motif. Cell Growth Different., 4: 57-65.

Pleasance, E.D., R.K. Cheetham, P.J. Stephens, D.J. McBride and S.J. Humphray et al., 2010. A comprehensive catalogue of somatic mutations from a human cancer genome. Nature, 463: 191-196. DOI: $10.1038 /$ nature 08658 .

Sandoval, J. and M. Esteller, 2012. Cancer epigenomics: Beyond genomics. Curr. Opin. Genet. Dev., 22: 50-55. DOI: 10.1016/j.gde.2012.02.008

Santolla, M.F., S. Avino, M. Pellegrino, E.M. De Francesco and P. De Marco et al., 2015. SIRT1 is involved in oncogenic signaling mediated by GPER in breast cancer. Cell Death and Disease, 6: e1834-e1834. DOI: $10.1038 /$ cddis.2015.201

Scorilas, A., T. Trangas, J. Yotis, C. Pateras and M. Talieri, 1999. Determination of c-myc amplification and overexpression in breast cancer patients: Evaluation of its prognostic value against c-erbB-2, cathepsin-D and clinicopathological characteristics using univariate and multivariate analysis. British J. Cancer, 81: 1385-1391. DOI: $10.1038 /$ sj.bjc.6693404

Semenza, G.L., 2010. Defining the role of hypoxiainducible factor 1 in cancer biology and therapeutics. Oncogene, 29: 625-634.

DOI: $10.1038 /$ onc. 2009.441

Sharma, S., T.K. Kelly and P.A. Jones, 2010. Epigenetics in cancer. Carcinogenesis, 31: 27-36. DOI: $10.1093 /$ carcin/bgp220

Sidransky, D., T. Mikkelsen, K. Schwechheimer, M.L. Rosenblum and W. Cavanee et al., 1992. Clonal expansion of p53 mutant cells is associated with brain tumour progression. Nature, 355: 846-847. DOI: $10.1038 / 355846 a 0$

Slee, E.A., D.J. O'Connor and X. Lu, 2004: To die or not to die: How does p53 decide? Oncogene, 23: 2809-2818. DOI: $10.1038 /$ sj.onc. 1207516

Talks, K.L., H. Turley, K.C. Gatter, P.H. Maxwell and C.W. Pugh et al., 2000. The expression and distribution of the hypoxia-inducible factors HIF1alpha and HIF-2alpha in normal human tissues, cancers and tumor-associated macrophages. Am. J. Pathol., 157: 411-421. DOI: $10.1016 / \mathrm{S} 0002-9440(10) 64554-3$

Tas, F., H. Hanse, A. Belce, S. Ilvan and A. Argon et al., 2005. Oxidative stress in breast cancer. Med. Oncol., 22: 11-15. DOI: 10.1385/MO:22:1:011 
Teng, Y., H. Jing, P. Aramsangtienchai, B. He and S. Khan et al., 2014. Efficient demyristoylase activity of SIRT2 revealed by kinetic and structural studies. Scientific Rep., 5: 8529-8529. DOI: $10.1038 /$ srep08529

Tsanou, E., E. Ioachim, E. Briasoulis, K. Damala and A. Charchanti et al., 2004. Immunohistochemical expression of Superoxide Dismutase (MnSOD) antioxidant enzyme in invasive breast carcinoma. Hist. Histopathol., 19: 807-13.

Vaquero, A., M.B. Scher, D.H. Lee, A. Sutton and H.L. Cheng et al., 2006. SirT2 is a histone deacetylase with preference for histone H4 Lys 16 during mitosis. Genes Dev., 20: 1256-1261. DOI: $10.1101 / \mathrm{gad} .1412706$

Wang, R.H., K. Sengupta, C. Li, H.S. Kim and L. Cao et al., 2008. Impaired DNA damage response, genome instability and tumorigenesis in SIRT1 mutant mice. Cancer Cell, 14: 312-23. DOI: 10.1016/j.ccr.2008.09.001

Weisz, L. A. Damalas, M. Liontos, P. Karakaidos and G. Fontemaggi et al., 2007. Mutant p53 enhances nuclear factor kappaB activation by tumor necrosis factor alpha in cancer cells. Cancer Res., 67: 2396-2401. DOI: 10.1158/0008-5472.CAN-06-2425
Wilking, M.J. and N. Ahmad, 2015. The Role of SIRT1 in Cancer. Am. J. Pathol., 185: 26-28. DOI: $10.1016 /$ j.ajpath.2014.10.002

Xue, W., L. Zender, C. Miething, R.A. Dickins and E. Hernando et al., 2007. Senescence and tumour clearance is triggered by $\mathrm{p} 53$ restoration in murine liver carcinomas. Nature, 445: 656-660. DOI: 10.1038 /nature05529

Yan, S.M., X. Han, P.J. Han, H.M. Chen and L.Y. Huang et al., 2014. SIRT3 is a novel prognostic biomarker for esophageal squamous cell carcinoma. Med. Oncol., 31: 103-103. DOI: $10.1007 / \mathrm{s} 12032-014-0103-8$

Yuan, H., L. Su and W.Y. Chen, 2013. The emerging and diverse roles of sirtuins in cancer: A clinical perspective. Onco. Targets Ther., 6: 1399-1416. DOI: $10.2147 /$ OTT.S37750

Zhang, D., J. Li, M. Costa, J. Gao and C. Huang, 2010. JNK1 mediates degradation HIF-1alpha by a VHLindependent mechanism that involves the chaperones Hsp90/Hsp70. Cancer Res., 70: 813-823. DOI: 10.1158/0008-5472.CAN-09-0448 